\title{
Dual-Energy 4-Phase CT Scan in Primary Hyperparathyroidism
}

W e read with interest the article by Gafton et $\mathrm{al}^{1}$ published in 2012. The authors illustrated the use of a dual-phase CT protocol for the enhancement characterization of parathyroid lesions and suggested the elimination of precontrast and delayed scan phases from 4D CT to reduce radiation exposure. In this letter, we describe the alternative approach of our institution to 4D CT. Our modified 4D CT protocol uses virtual noncontrast (VNC) images obtained from dual-energy CT-reconstructed venous-enhanced images to replace true precontrast scans. This process allows a complete examination of the contrast enhancement and washout characteristics of parathyroid adenomas on 4D $\mathrm{CT}$, without reducing the number of scan phases.

Here we present a 57-year-old female patient who was suspected of having primary hyperparathyroidism (PHPT) and was scanned by using our modified 4D CT protocol. The institutional review board approval was waived for this dose-saving study. The patient was referred for neck imaging using our modified protocol after negative technetium Tc99m-sestamibi scintigraphy findings, despite the presence of elevated total serum calcium and parathyroid hormone levels. A modified 4-phase CT scan was obtained, with a dual-energy scan acquired during the venous phase.

\section{Image Acquisition and Processing}

All scans were obtained with the patient in the supine position by using a 64-section dual-source CT scanner (Somatom Definition; Siemens, Erlangen, Germany) to acquire contiguous axial images from the lower margin of the mandible to the level of the inferior aortic arch. Intravenous injection of $50 \mathrm{~mL}$ of iodinated contrast material (iohexol, Omnipaque, 350 mg/mL; GE Healthcare, Milwaukee, Wisconsin), followed by $50 \mathrm{~mL}$ of saline chaser bolus, was administered via the right antecubital vein at a rate of $3 \mathrm{~mL} / \mathrm{s}$ by using a 20 -gauge catheter. Scan acquisition in the arterial, dualenergy venous, and delayed phases was performed at 25,55 , and 85 seconds, respectively. Single-energy CT in the arterial and delayed phases was performed by using a collimation of $16 \times 0.75$ $\mathrm{mm}$ and a pitch value of 1 at $120 \mathrm{kV}$ (peak)/62 mAs, whereas dual-energy CT was performed in the venous phase by using a $32 \times 0.6 \mathrm{~mm}$ collimation, a pitch value of 1 , and tube current

http://dx.doi.org/10.3174/ajnr.A3657 settings of $80 \mathrm{kVp} / 210 \mathrm{mAs}$ (tube A) and $140 \mathrm{kVp} / 50 \mathrm{mAs}$ (tube B). Both single- and dual-energy CT scans were obtained with a gantry rotation speed of 0.8 seconds, with on-line tube current modulation (Care Dose 4D; Siemens). The estimated CT dose index and dose-length product were recorded for the scan session.

All images were subsequently reconstructed into axial and sagittal views with a section thickness of $3 \mathrm{~mm}$ and an increment of 3 $\mathrm{mm}$, with an FOV of $20 \mathrm{~cm}$ and a $512 \times 512$ matrix, by using a B30f kernel for single-energy CT and a D30f kernel for dual-energy CT on a syngo MultiModality Workplace (MMWP), Version VE 31A (Siemens). The dual-energy venous raw data from both detectors were reconstructed into $80 \mathrm{kV}$ (peak), $140 \mathrm{kVp}$, and weighted average $120 \mathrm{kVp}$ images (30\% of the image information was derived from the $80 \mathrm{kVp}$ image and $70 \%$ from the $140 \mathrm{kVp}$ image). Further postprocessing to the dual-energy venous dataset by using the Liver Virtual Unenhanced application on the syngo MMWP was performed to generate the iodine map from the individual CT voxels, as well as the subtraction of the iodine signal from the dataset to produce a VNC-enhanced image.

\section{Image Analysis}

All images were analyzed on syngo MMWP. Circular regions of interest were defined and positioned within the center of the parathyroid adenoma to measure the contrast attenuation values. The size was chosen as approximately $5.0 \mathrm{~mm}^{2}$ to cover almost the entire lesion but avoiding beam-hardening and partial volume artifacts at the borders of the lesion. The mean \pm SD values in Hounsfield units measured from the respective images acquired from each scan phase were recorded. Results from the modified 4D CT scan are shown in Figs 1-3. A subsequent directed parathyroidectomy revealed the presence of a parathyroid adenoma (Figs 1-3).

Our findings suggest that VNC images could potentially replace true precontrast images to provide an accurate differentiation of the parathyroid adenoma from surrounding normal tissues, by characterizing its contrast enhancement with time. It has been reported in the literature that VNC images can provide a good approximation of the attenuation values similar to that in true precontrast images and, accordingly, lower radiation exposure in patients by omitting the need for precontrast scanning. ${ }^{2}$ The resulting images obtained by using 


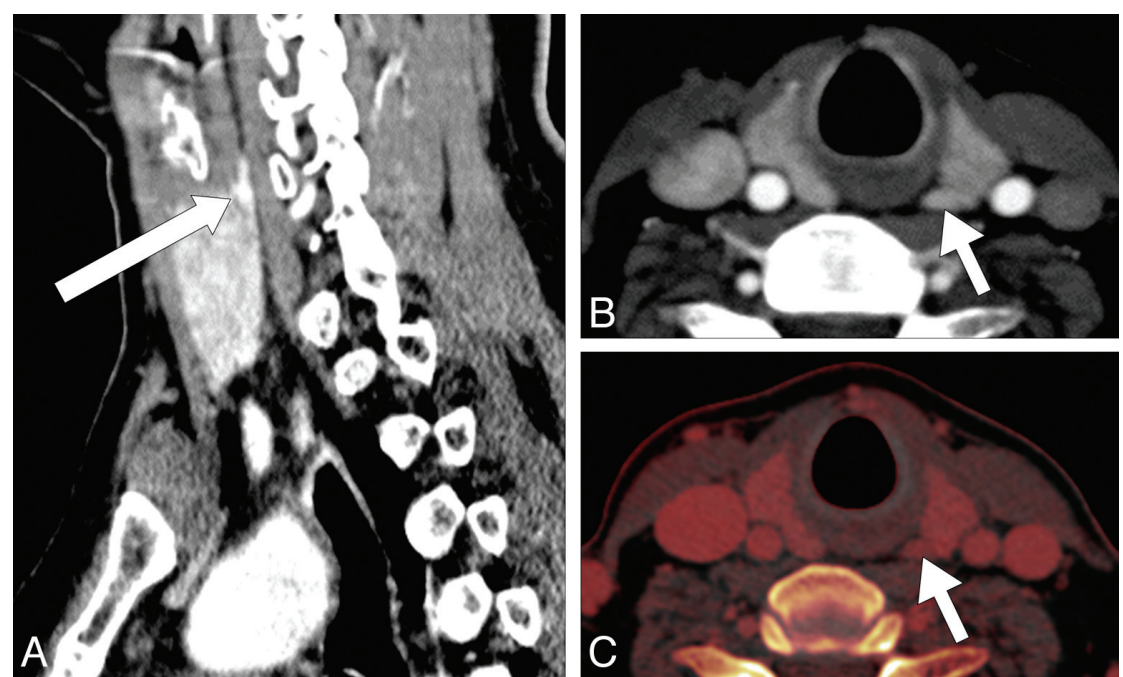

FIG 1. CT examination of the patient by using our modified 4D CT protocol shows a $6 \times 3 \mathrm{~mm}$ nodular parathyroid adenoma (arrows) lying in the superomedial aspect, posterior to the left thyroid lobe in both sagittal $(A)$ and axial $(B)$ sections. The iodine overlay image (acquired by using dual-energy $C T$ in the venous phase), also obtained by using this protocol, allows the measurement of iodine concentration in the tissues, to differentiate between the parathyroid adenoma and surrounding thyroid tissues $(C)$. Directed parathyroidectomy and histopathologic examination, thereafter, confirmed the presence of a parathyroid adenoma. tion in the overall radiation exposure by approximately $20 \%$ (Tables 1 and 2 ). In addition, with appropriate software (which we do not have), measurement of the tissue iodine concentration (in milligrams/deciliter) is also possible on the iodine overlay image. This has potentially more useful implications in head and neck imaging than conventional 4D CT because it allows the radiologist to determine whether the lesion is arising from thyroid tissues or is extrathyroidal in nature.

In our experience, provision of images from all 4 phases, including the precontrast and delayed scans, is helpful in determining the baseline attenuation value and dynamic changes with time. Various authors have proposed modified 4D CT methods, mostly by eliminating 1 or 2 phases from the scan technique. ${ }^{1,3}$ These may reduce the diagnostic confidence of the reporter. We feel that the provision of our modified protocol provided a reasonably good diagnostic anatomic localization of the parathyroid adenoma (Fig 1). A complete 4-phase examination of the enhancement characteristics of the parathyroid adenoma (rapid contrast uptake and washout) was feasible (Figs 2 and 3), with an estimated reduc-

4 phases is crucial for the localization of small or ectopic parathyroid adenoma or lesions situated in crowded structures in the neck or other distal locations such as the mediastinum. ${ }^{4,5}$

However, one drawback in using this modified technique is the subtraction of iodine attenuation from the thyroid glands on VNC images, thereby possibly negating the potential benefit of this modified $4 \mathrm{D}$ CT study. Admittedly, a true precontrast scan does confer additional information of soft-tissue contrast between the parathyroid adenoma and surrounding soft tissue (thyroid tissue is inherently denser compared with adenomatous tissue, due to its high iodine concentration); this, however, comes with added radiation burden. In the authors' experience, careful analysis of the VNC, arterial, venous, and delay phases can help in separating parathyroid adenoma tissues from normal surrounding soft tissues (such as the thyroid gland). In addition, for patients with PHPT and concurrent thyroiditis, in which the attenuation of the thyroid gland is low to begin with, having an iodine overlay map allows the quantification of iodine concentration of a particular lesion, enabling accurate lesion characterization.

In conclusion, we believe our modified dual-energy protocol can allow reduction in radiation exposure during 4D CT parathyroid scanning without compromising the need for studying the enhancement characteristics of these lesions. Nevertheless, further studies 

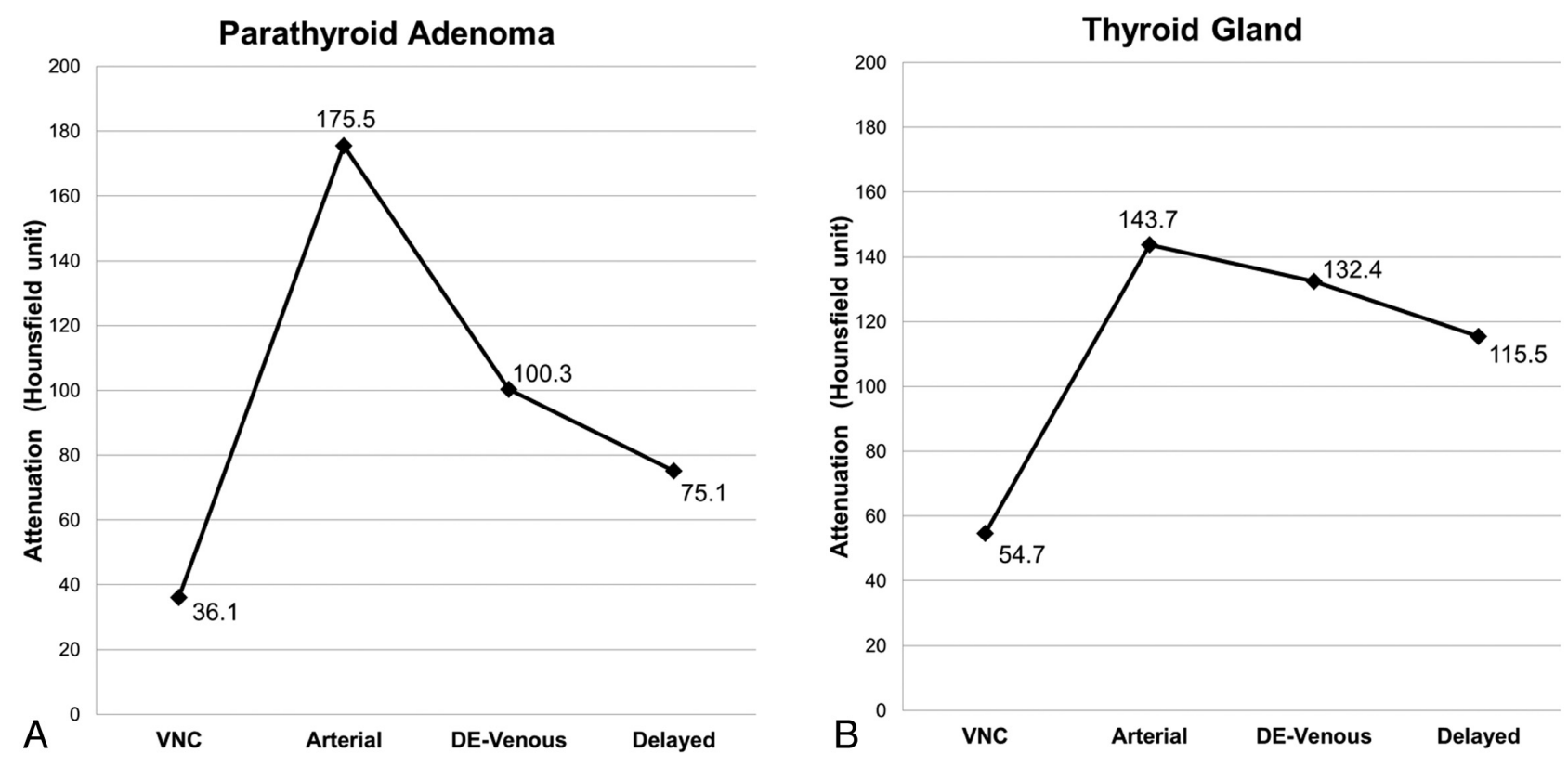

FIG 3. Attenuation plots show the characteristic dynamic contrast-enhancement pattern of the parathyroid adenoma (ie, rapid contrast uptake and washout, $(A)$ and a normal-functioning right thyroid gland $(B)$ measured from the same scans obtained from the patient diagnosed with PHPT by using our modified 4D CT protocol. The mean Hounsfield unit attenuations of the imaged structures in the VNC (reconstructed from the dual-energy venous [DE-venous]), arterial (by using bolus tracking), DE-venous ( 55 seconds), and delayed ( 85 seconds) phases were measured by using region-of-interest analysis as shown in Fig 2.

Table 1: Standard 4D CT protocol in another patient ${ }^{\mathrm{a}}$

\begin{tabular}{lcc}
\hline & \multicolumn{2}{c}{ Measurement } \\
\cline { 2 - 3 } \multicolumn{1}{c}{ Phase } & CTDI $_{\text {vol }}$ (mGy) & DLP (mGy.cm) \\
\hline Precontrast & 27.16 & 452 \\
Arterial & 27.32 & 475 \\
Venous & 27.08 & 471 \\
Delayed & 27.32 & 475 \\
Total & 108.88 & 1873 \\
\hline
\end{tabular}

Note:- $C T D I_{\text {vol }}$ indicates $C T$ dose index volume; DLP, dose-length product.

a These measurements obtained during our modified $4 \mathrm{D} C \mathrm{C}$ scan on another weightand sex-matched patient were compared with values obtained during standard 4D CT (including the precontrast scan) of our patient. They showed an estimated $20 \%$ reduction in the radiation exposure.

Table 2: Modified 4D CT protocol in our current patient ${ }^{\mathrm{a}}$

\begin{tabular}{|c|c|c|}
\hline \multirow[b]{2}{*}{ Phase } & \multicolumn{2}{|c|}{ Measurement } \\
\hline & $\begin{array}{l}\text { CTDI }_{\text {vol }} \\
\text { (mGy) }\end{array}$ & $\begin{array}{c}\text { DLP } \\
\text { (mGy.cm) }\end{array}$ \\
\hline Arterial & 26.92 & 475 \\
\hline DE-venous ${ }^{\mathrm{b}}$ & 31.78 & 583 \\
\hline Delayed & 26.68 & 471 \\
\hline Total & 85.38 & 1529 \\
\hline \% Reduction from standard protocol & 21.6 & 18.4 \\
\hline
\end{tabular}

Note:- CTDI $_{\text {vol }}$ indicates $C T$ dose index volume; DLP, dose-length product; DE, dual-energy.

${ }^{a}$ These measurements obtained during our modified $4 D C T$ scan on the current patient were compared with values obtained during standard $4 \mathrm{DCT}$ (including the precontrast scan) of another weight- and sex-matched patient. Both CTDI ${ }_{\text {vol }}$ and DLP measurements were recorded during modified $4 \mathrm{D} C \mathrm{CT}$ (excluding the precontrast scan) for the current patient (61.6-kg female patient) illustrated in this case presentation. They showed an estimated $20 \%$ reduction in the radiation exposure.

${ }^{\mathrm{b}}$ The venous phase scan was acquired using dual-energy CT (DE-venous) to obtain the VNC image. should be conducted to validate the real clinical impact of our suggested protocol for diagnosing PHPT in a larger group of patients.

\section{ACKNOWLEDGMENTS}

We thank the Singapore General Hospital Department of Radiology for support of this study and the technologists Muhammad Illyyas and Joanne Chia for performing the scan and image reconstruction.

\section{REFERENCES}

1. Gafton AR, Glastonbury CM, Eastwood JD, et al. Parathyroid lesions: characterization with dual-phase arterial and venous enhanced CT of the neck. AJNR Am J Neuroradiol 2012;33:949-52

2. Tawfik AM, Kerl JM, Razek AA, et al. Image quality and radiation dose of dual-energy CT of the head and neck compared with a standard 120-kVp acquisition. AJNR Am J Neuroradiol 2011;32:1994-99

3. Kutler DI, Moquete R, Kazam E, et al. Parathyroid localization with modified 4D-computed tomography and ultrasonography for patients with primary hyperparathyroidism. Laryngoscope 2011; 121:1219-24

4. Hunter GJ, Schellingerhout D, Vu TH, et al. Accuracy of four-dimensional CT for the localization of abnormal parathyroid glands in patients with primary hyperparathyroidism. Radiology 2012;264: 789-95

5. Chazen JL, Gupta A, Dunning A, et al. Diagnostic accuracy of 4D-CT parathyroid adenomas and hyperplasia. AJNR Am J Neuroradiol 2012;33:429-33 Singapore General Hospital Singapore 